\title{
STK11 is required for the normal program of ciliated cell differentiation in airways
}

\author{
Qiqi Chu ${ }^{1,2}$, Changfu Yao ${ }^{3}$ Xiangbing Qi $\mathbb{C}^{2}$, Barry Raymond Stripp ${ }^{3}$ and Nan Tang ${ }^{2,4}$
}

\begin{abstract}
The functional properties of mucosal surfaces are dependent on establishing the correct proportions of specialized epithelial cell types. Multiciliated cells (also known as ciliated cells) are evolutionarily conserved and functionally indispensable epithelial cells, as suggested by the link between ciliated cell dysfunction and chronic human disease. Ciliated cell differentiation is an ordered process that involves initial cell fate determination and multiciliogenesis. STK11, a serine/threonine kinase, has been reported to be downregulated in human diseases associated with ciliopathies and functions as a tumor suppressor. Here, we show that STK11 is a physiological factor for the normal program of ciliated cell differentiation by phosphorylating MARK3, which directly suppresses ERK1/2 mediated pRB inactivation. Loss of Stk 11 in airway progenitors impairs the differentiation of ciliated cells in both embryonic and adult airways. Our study establishes that STK11/MARK3/ERK1/2 signaling cascade is a key regulator to integrate ciliated cell fate commitment and the subsequent process of multiciliogenesis.
\end{abstract}

\section{Introduction}

The airway epithelium serves critical functions to protect the lung from environmental insults. Two major types of epithelial cells in the airway epithelium, secretory and multiciliated cells (also known as ciliated cells), provide airway secretions and ensure removal of invading pathogens and inhaled particles ${ }^{1}$. It is known that the balance between secretory and ciliated cells is fundamental for normal airway function. Impaired differentiation or abnormal functions of secretory and/or ciliated cells lead to many respiratory diseases, including airway infections, cystic fibrosis lung disease, and chronic obstruction pulmonary disease ${ }^{1}$. Therefore, the differentiation of secretory and ciliated cells needs to be tightly regulated during development, homeostasis, and regeneration.

Correspondence: Barry Raymond Stripp (Barry.Stripp@cshs.org) or

Nan Tang (tangnan@nibs.ac.cn)

${ }^{1}$ College of Life Sciences, Beijing Normal University, 100875 Beijing, China

${ }^{2}$ National Institute of Biological Sciences, 102206 Beijing, China

Full list of author information is available at the end of the article.

These authors contributed equally: Qiqi Chu, Changfu Yao
Ciliated cells are derived from multipotent endodermal progenitor cells of the embryonic lung and either secretory or basal progenitors of the postnatal airway ${ }^{2}$. Seminal work has demonstrated that Notch signaling, an evolutionarily conserved cell fate determinant, plays a key role in controlling the balance between secretory versus ciliated cell fates in both embryonic and adult lungs ${ }^{3-10}$. Activation of Notch signaling results in more secretory cells $^{4,6,8,10}$, while inhibition results in an increased number of ciliated cells and a decreased number of secretory cells $^{3,4,6,9,11}$. It is known that in response to cell growth and cytokine signaling, Notch also works with a significantly more complex regulatory network, including p53 and STAT3 signaling, to control epithelial cell differentiation ${ }^{11,12}$. In addition to the function of these signaling networks in ciliated cell fate specification, multiple genes, such as Multicillin and E2F4, have been shown to regulate a subsequent multiciliogenesis process by inducing expression of centriole biogenesis genes and promoting centriole assembly ${ }^{13-15}$. However, the mechanisms that integrate these factors to coordinate the step-by-step process of ciliated cell differentiation remain largely unknown.

\section{(c) The Author(s) 2019}

(c) (i) Open Access This article is licensed under a Creative Commons Attribution 4.0 International License, which permits use, sharing, adaptation, distribution and reproduction cc) in any medium or format, as long as you give appropriate credit to the original author(s) and the source, provide a link to the Creative Commons license, and indicate if changes were made. The images or other third party material in this article are included in the article's Creative Commons license, unless indicated otherwise in a credit line to the material. If material is not included in the article's Creative Commons license and your intended use is not permitted by statutory regulation or exceeds the permitted use, you will need to obtain permission directly from the copyright holder. To view a copy of this license, visit http://creativecommons.org/licenses/by/4.0/. 
Here, using single-cell RNA-sequencing (scRNA-seq) analysis to characterize ciliated cell differentiation in adult airways, we found that the tumor suppressor gene $S t k 11$ is simultaneously induced at the time of ciliated cell fate determination. STK11, a serine/threonine kinase, has been reported to be downregulated in human diseases associated with ciliopathies, including Bardet-Biedl syndrome $^{16}$, Polycystic kidney disease ${ }^{17}$, and Nephronophthisis ${ }^{18}$. We found here that Stk11 was highly enriched in ciliated cells in both embryonic and adult lungs, and deleting Stk11 specifically from epithelial progenitor cells impairs ciliated cell differentiation in both embryonic and adult lungs. We have demonstrated that a STK11/MARK3/ERK1/2 signaling cascade acts to enforce ciliated cell lineage commitment and multiciliogenesis in embryonic and adult airways.

\section{Results}

The expression of Stk11 is associated with the differentiation of ciliated cells in the airway epithelium

To characterize the differentiation program of ciliated cells, we isolated $\mathrm{EpCAM}^{+} \mathrm{CD} 45^{-} \mathrm{CD} 31^{-}$intropulmonary epithelial cells from the adult mouse lungs and performed scRNA-seq analysis. In total, 5842 epithelial cells were used for integrating scRNA-seq analysis. Six cell clusters were identified by the expression of known marker genes, including alveolar type II cells; alveolar type I cells; proximal club cells (Prox-Club) ${ }^{19}$; distal club cells (DisClub) ${ }^{19}$; cells that express both Scgb1a1 and Foxj1 (Club/ Ciliated); and ciliated cells (Ciliated) (Supplementary Fig. S1a).

To better characterize the differentiation program of ciliated cells, we extracted 1767 cells in Prox-Club, DisClub, Club/Ciliated, and Cililated clusters and performed further analyses (Fig. 1a). Interestingly, we found that Stk11, which was well known for its function as a tumor suppressor frequently mutated in nonsmall cell lung cancer, was abundantly expressed in cells within the Club/ Ciliated and Ciliated cell clusters ${ }^{20}$ (Fig. 1a, Supplementary Fig. S1b). We further described the trajectory of ciliated cell differentiation using a diffusion map and characterized the pseudo-temporal expression levels of genes during ciliated cell differentiation (Fig. 1b). We found that the expression level of Stk11 simultaneously increased during ciliated cell differentiation process (Fig. $1 b)$.

The decreased expression level of Stk11 has been reported in human ciliopathies ${ }^{16-18}$. By calculating the enrichment score of Stk11 in different cell clusters, we found that Stk11 is highly enriched in cells of both Club/ Ciliated and Ciliated clusters in adult lungs (Fig. 1c). By immunofluorescence staining with antibodies against acetylated- $\alpha$-tubulin and STK11, we found that STK11 was expressed among many airway epithelial cells of embryonic day (E) 16.5 lungs, including a majority of acetylated- $\alpha$-tubulin $^{+}$ciliated cells and some undiffer-

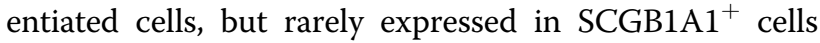
(Fig. 1d, Supplementary Fig. S1c). Together, these results suggest that Stk11 may function in regulating the differentiation of ciliated cells.

\section{Deletion of Stk11 in endodermal progenitors of embryonic lung or secretory progenitors of adult lung impairs ciliated cell differentiation}

To explore the potential roles of STK11 in ciliated cell differentiation of the developing airway, Shh-Cre; $S t k 11^{\mathrm{F} / \mathrm{F}}$ mice (Shh-Stk11) were generated, in which Stk11 is specifically deleted in endodermal progenitors of the embryonic lung epithelium. In the developing mouse airway, ciliated cells are generated from endodermal progenitor cells. $\mathrm{MYB}^{+}$ciliated cells appear between E13.5 and E14.5, and express the transcription factor $\mathrm{FOXJ}^{+}$as they differentiate into mature ciliated cells ${ }^{21}$. Epithelial cell differentiation was evaluated in lungs of E16.5 Shh-Stk11 and Shh-Cre; Stk11 ${ }^{\mathrm{F} /+}$ (Control) mice by immunofluorescence. The abundance of $\mathrm{FOXJ} 1^{+}$and $\mathrm{MYB}^{+}$ciliated cells were each significantly decreased, whereas the percentage of SCGB1A1 ${ }^{+}$club cells was significantly increased, in both tracheal and intrapulmonary epithelia of E16.5 Shh-Stk11 mice, as compared to their littermate Controls (Fig. 2a, b, Supplementary Fig. S2a-d). Notably, excluding the possibility that the impaired ciliated cell differentiation in the Shh-Stk11 lungs results from a developmental delay, we observed similar changes in E18.5 and E20.5 Shh-Stk11 lungs as compared to Control lungs (Supplementary Fig. S2e-h).

During embryonic lung development, endodermal progenitors give rise not only to club and ciliated cells but also to basal and neuroendocrine cells. Therefore, we further analyzed the differentiation of basal cells and neuroendocrine cells in the epithelium of E16.5 Shh-Stk11 and Control lungs. The proportion of basal cells (as a function of total tracheal epithelia) was not significantly different between lungs of Shh-Stk11 and Control embryos (Supplementary Fig. S2i, j). In addition, we observed the proportion of neuroendocrine cells was not significantly different in both genotypes of lungs (Supplementary Fig. S2k, l). These results establish that the loss of Stk11 does not significantly affect the differentiation of basal cells or neuroendocrine cells in embryonic lungs.

We further investigated the effect of conditionally deleting Stk11 in $\mathrm{SOX}^{+}$airway progenitor cells. It has been shown that SOX2 is expressed exclusively in airway progenitor cells during embryonic lung development ${ }^{22}$. Pregnant females carrying Sox2-CreER; Stk $11^{\mathrm{F} / \mathrm{F}}$; Rosa26mTmG (Sox2-Stk11) and Sox2-CreER; Stk11 ${ }^{\mathrm{F} /+}$; Rosa26- 

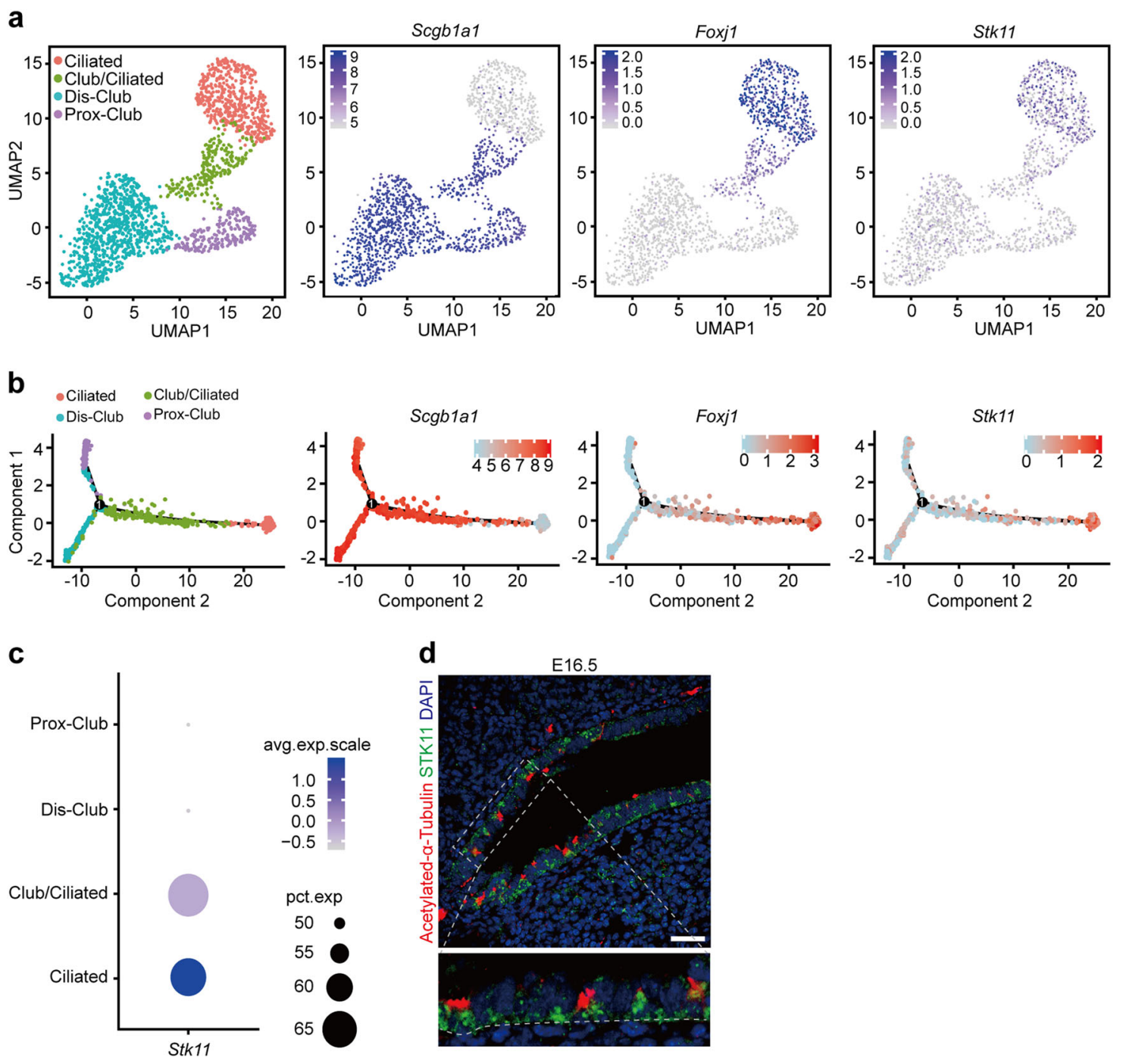

Fig. 1 The expression level of Stk11 is associated with the differentiation of ciliated cells in airways. a The UMAP plots of scRNA-seq analyses show the expression of Scgb1a1, Foxj1, or Stk11 in adult airway epithelial cells from adult lungs. $\mathbf{b}$ The pseudo-time trajectories show that the expression level of Scgbla1 decreased during the ciliated cell differentiation process, whereas the expression levels of Foxj1 and Stk11 increased during the ciliated cell differentiation process. $\mathbf{c}$ The dot plot of Stk11 expression score in different cell types. The dot size represents the proportion of cells in a cluster that express the gene. The dot color corresponds to the average expression level of the gene. $\mathbf{d}$ E16.5 lungs were stained with antibodies against acetylated-a-Tubulin and STK11. Scale bars: $25 \mu \mathrm{m}$

mTmG (Control) embryos were injected with tamoxifen at E13.5 (Fig. 2c), a stage at which the $\mathrm{SOX}^{+}$airway progenitor cells have not started to differentiate into club or ciliated cells. At E16.5, we found that the proportion of FOXJ $1^{+}$ciliated cells was significantly decreased and that the proportion of SCGB1A1 $1^{+}$club cells was significantly increased among lineage-labeled intrapulmonary airway epithelial cells in Sox2-Stk11 lungs as compared to those of Control embryos (Fig. 2d, e).

Club cells in the adult airway function as secretory progenitor cells that can self-renew and slowly differentiate into ciliated cells to maintain the correct proportions of specialized epithelial cell types ${ }^{12,23,24}$. We next sought to investigate the role of STK11 in regulating ciliated cell differentiation in the adult airway. To achieve this, Scgb1a1-CreER; Stk11 ${ }^{\mathrm{F} / \mathrm{F}}$; Rosa26mTmG (Scgb1a1-Stk11) adult mice were injected with three doses of tamoxifen for conditional deletion of Stk11 within airway club cells and were monitored for 30 days to determine the impact on ciliated cell differentiation (Fig. 2f). The percentage of newly differentiated ciliated cells among lineage-labeled cells decreased significantly in Scgb1a1-Stk11 mice (Fig. 2g, h). These results together with those for embryonic lung support a role for STK11 in controlling ciliated cell differentiation. 


\section{a}

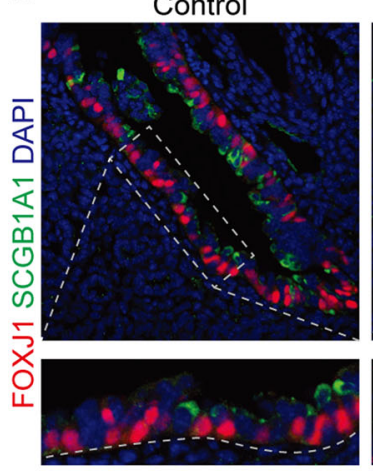

C

Sox2-CreER; Rosa26-mTmG; Stk11 ${ }^{\text {F/+ }}$ (Control)

Sox2-CreER; Rosa26-mTmG; Stk11F/F (Sox2-Stk11)

Inject tamoxifen

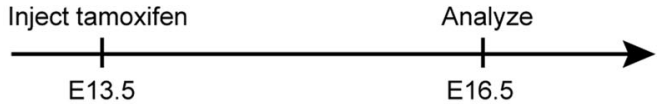

d

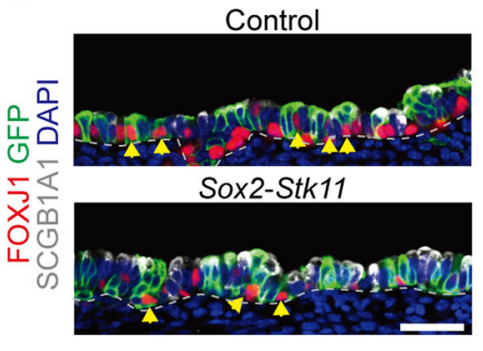

b

Control $\square$ Shh-Stk11

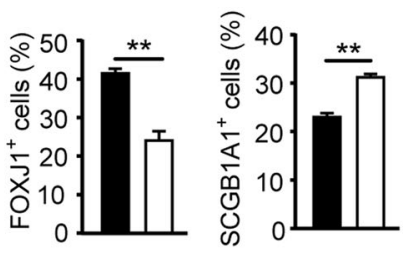

f

Scgb1a1-CreER; Rosa26-mTmG; Stk11 ${ }^{\mathrm{F} /+}$ (Control) Scgb1a1-CreER; Rosa26-mTmG; Stk11FIF (Scgb1a1-Stk11)

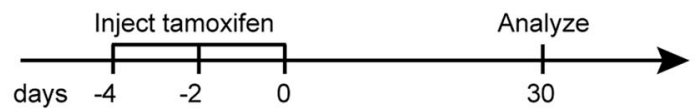

g

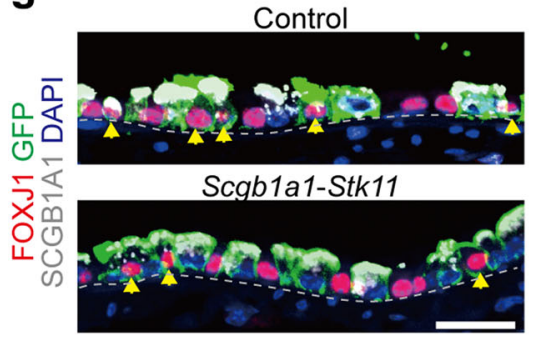

h

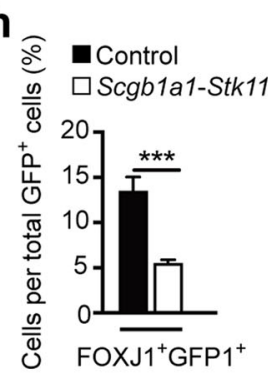

Fig. 2 Loss of Stk11 in airway progenitor cells impairs ciliated cell differentiation. a E16.5 lungs were stained with antibodies against FOXJ1 and SCGB1A1. b The proportion of FOXJ1 ${ }^{+}$cells (Left, $n=3$ ) and SCGB1A $1^{+}$cells (Right, $n=4$ ) in the intrapulmonary airways of E16.5 lungs. c Pregnant mice carrying Sox2-CreER; Rosa26-mTmG; Stk11 ${ }^{\mathrm{F} /+}$ (Control) and Sox2-CreER; Rosa26-mTmG; Stk11 ${ }^{\mathrm{F} / \mathrm{F}}$ (Sox2-Stk11) embryos were treated with tamoxifen at E13.5 and analyzed at E16.5. d E16.5 lungs were stained with antibodies against GFP, FOXJ1, and SCGB1A1. The yellow arrowheads indicate cells expressing both GFP and FOXJ1. e The proportion of FOXJ1 ${ }^{+} \mathrm{GFP}^{+}$cells to GFP ${ }^{+}$cells (Left) (Control, $n=6$; Sox2-Stk $11, n=5$ ) and SCGB1A1 ${ }^{+} \mathrm{GFP}^{+}$cells to GFP ${ }^{+}$cells (Right) $(n=3)$ in the intrapulmonary airways of E16.5 lungs. $\mathbf{f}$ Ten-week-old mice were treated with three doses of tamoxifen and analyzed after 30 days. $\mathbf{g}$ Lungs were stained with antibodies against GFP, FOXJ1, and SCGB1A1 at day 30. The yellow arrowheads indicate cells expressing both GFP and FOXJ1. $\mathbf{h}$ The proportion of FOXJ1 ${ }^{+} \mathrm{GFP}^{+}$cells to GFP ${ }^{+}$cells at day $30(n=4)$. ${ }^{*} P<0.05 ;{ }^{* *} P<0.01$; ${ }^{* * *} P<0.001$. Data shown in the graphs are means \pm SEM. Student's $t$ test. Scale bars: $\mathbf{a}, \mathbf{d}: 25 \mu \mathrm{m}, \mathbf{g}: 20 \mu \mathrm{m}$ 
The cell-cycle exit and ciliated cell differentiation are controlled by STK11 within embryonic and adult airways

To further explore the mechanisms through which STK11 regulates ciliated cell differentiation, RNA-seq was performed to compare the transcriptomes of E16.5 ShhStk11 and Control mice. Consistent with our immunostaining results, we found that ciliated cell markers (e.g., Mcidas, Myb, and $F o x j 1^{25}$ ) were significantly reduced and found that secretory cell markers, including a club cell marker Scgb1a1 and a mucous cell marker Muc5b, were significantly increased in the lungs of Shh-Stk11 embryos (Fig. 3a-c, Supplementary Dataset S1). We performed gene ontology (GO) analysis to identify the enriched terms amongst the differentially expressed genes in ShhStk11 lungs and also found that many of the significantly upregulated genes have known roles as positive regulators of cell proliferation and cell-cycle progression (e.g., $\operatorname{Ranbp2}^{26}, \operatorname{Smc5}^{27}, \mathrm{Mki67}^{28}, \operatorname{Sgo}^{2 a^{29}}$, Taf1 $^{30}, \mathrm{Ccnd2} 2^{31}$, and Cenpe $^{32}$ (Fig. 3a, b, Supplementary Fig. S3a, and Supplementary Dataset S1). Many of the significantly downregulated genes are known to participate in cilium movement and assembly (Fig. 3a, c, Supplementary Fig. S3b, and Supplementary Dataset S1). These results indicate that both ciliated cell differentiation and cell-cycle status are significantly affected in the Shh-Stk11 lungs.

We therefore analyzed the cell-cycle status of airway epithelial cells at E16.5 by propidium iodide (PI) staining, and found that $15.4 \%$ of the $\mathrm{EpCAM}^{+}$cells had entered the G2/M phase in Shh-Stk11 lungs, whereas only 11\% of the $\mathrm{EpCAM}^{+}$cells entered the G2/M phase in Control lungs (Supplementary Fig. S3c). Consistent with this PI staining result, the proportions of $\mathrm{PH}^{+}$(phospho-histone $\mathrm{H} 3$, a mitosis-specific marker) and $\mathrm{Ki}^{+} 7^{+}$intrapulmonary airway epithelial cells (Ki67 is a protein present during all active phases of the cell cycle; i.e., $G_{1}, S, G_{2}$, and mitosis) were significantly increased in Shh-Stk11 lungs compared to Control lungs (Supplementary Fig. S3d-g). In addition, loss of Stk11 does not induce apoptosis in embryonic lungs (Supplementary Fig. S3h). We also investigated the impact of STK11 on proliferation of epithelial cells in adult airways. Scgb1a1-Stk11 and Control mice were given IdU water for 30 days prior to sacrifice, and the cumulative IdU-labeling index was determined for lineagelabeled intrapulmonary airway epithelial cells (Supplementary Fig. S3i): there were significantly more $\mathrm{IdU}^{+}$ lineage-labeled epithelial cells in Scgb1a1-Stk11 mice than that in Control mice (Supplementary Fig. S3j, k).

\section{Inhibiting cell proliferation partially rescues the defective ciliated cell differentiation in airways of Stk11-deficient mice}

Considering that STK11 is known to suppress tumor growth by inhibiting cell proliferation ${ }^{33,34}$, we hypothesize that deletion of Stk11 either in endodermal progenitors of developing lung or in secretory progenitors of adult lung, prevents these progenitor cells from exiting an active cycling state that is necessary for ciliated cell differentiation. To test this, we determined whether inhibitors of the cell cycle could rescue the ciliated cell differentiation defect in lungs of Shh-Stk11 and/or Scgb1a1-Stk11 mice. Pregnant females carrying Shh-Stk11 and Control embryos were treated with PD0332991, a CDK4/6 inhibitor $^{35,36}$, or vehicle once daily via oral gavage from E12.5 to E14.5, and embryonic lungs collected at E16.5 for analysis (Fig. 3d). CDK4/6 are known to initiate the phosphorylation of $\mathrm{pRB}$, which disrupts its binding with E2Fs and allows cell-cycle entry ${ }^{37}$. We confirmed that the PD0332991 treatment could specifically reduce the number of p-pRB ${ }^{+}$cells in both the Control and ShhStk11 lungs (Supplementary Fig. S3l, m). Interestingly, we found that Control lungs had a much lower proportion of p-pRB ${ }^{+} \mathrm{Ki} 67^{+}$epithelial cells than did Shh-Stk11 lungs, indicating that the cell proliferation in the Stk11-wild-type epithelium is largely driven by p-pRB-independent regulatory mechanisms. Loss of Stk11 only activates p-pRBdependent cell-cycle progression (Supplementary Fig. S3n, o), demonstrating that STK11-dependent and STK11-independent regulation simultaneously occur in airway progenitor cells. Not surprisingly, the proliferative response of the intrapulmonary airway epithelium of ShhStk11 lungs was significantly attenuated by the PD0332991 treatment (Fig. 3e, f). Importantly, the proportion of ciliated cells in intrapulmonary airways of PD0332991-treated Shh-Stk11 embryos was significantly restored compared to those of vehicle-treated Shh-Stk11 embryos (Fig. 3g, h).

Next, we treated Scgb1a1-Stk11 mice with PD0332991 or vehicle once every other day for 30 days (Fig. 3i). PD0332991 treatment significantly decreased the proportion of $\mathrm{IdU}^{+}$lineage traced cells in Scgb1a1-Stk11 mice (Fig. 3j, k). Decreased proliferation in airways of PD0332991-treated Scgb1a1-Stk11 mice restored ciliated cell differentiation to levels observed in vehicle-treated Control intrapulmonary airways (Fig. 3l, m). Taken together, these results indicate that the inhibition of cellcycle progression in secretory progenitor cells of adult airways, a process regulated by STK11, allows the differentiation of ciliated cells.

\section{Ciliated cell differentiation requires the kinase activity of STK11}

STK11 is a serine/threonine protein kinase that controls the activity of multiple downstream kinases which fulfill diverse roles in embryonic development and adult tissue homeostasis $^{38-40}$. To understand the role of STK11 kinase activity in regulating ciliated cell differentiation, we generated recombinant adenoviral vectors expressing either wild-type STK11 (Ad-STK11 ${ }^{\mathrm{WT}}$ ) or kinase-dead STK11 


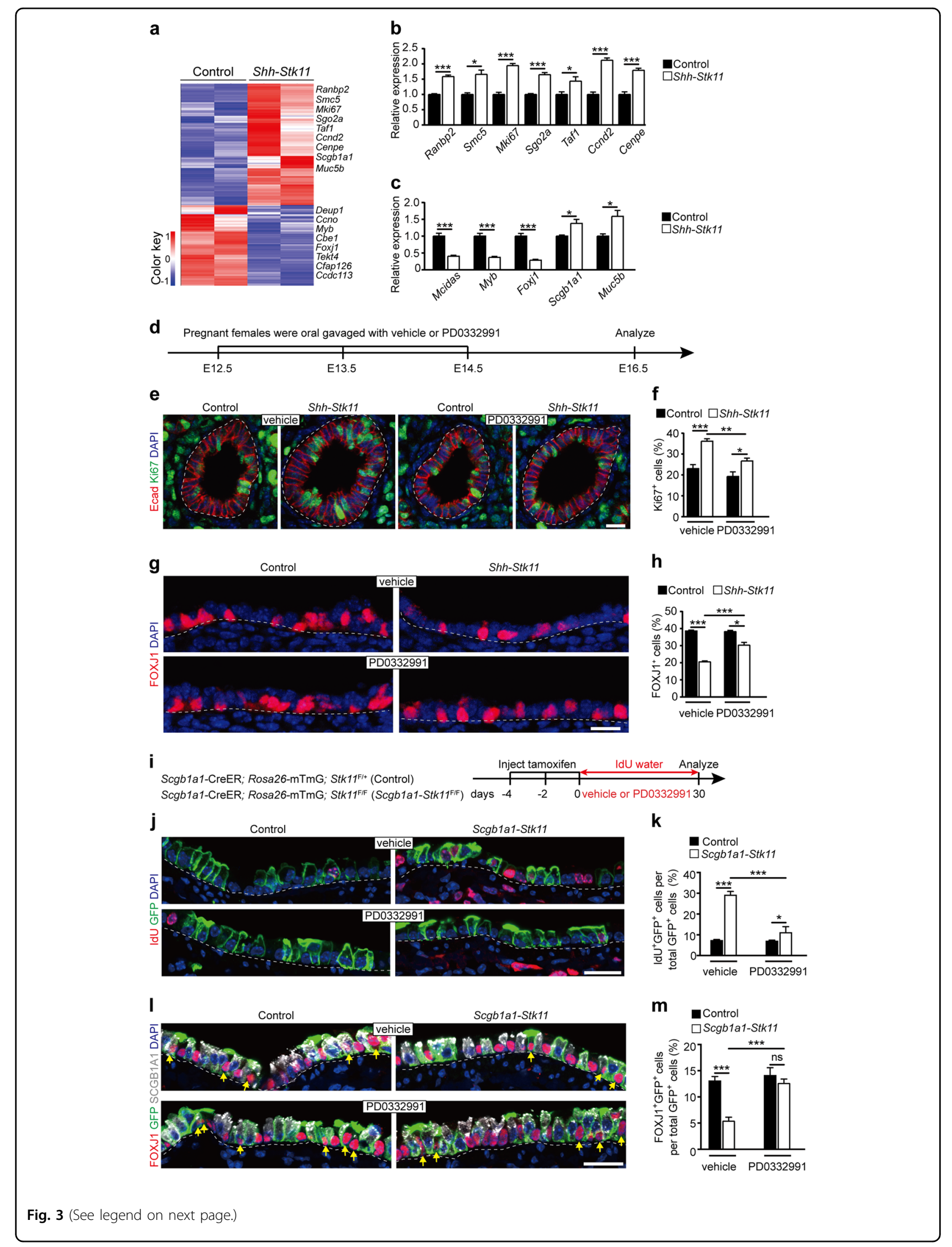


(see figure on previous page)

Fig. 3 Inhibiting cell proliferation partially rescues the impaired ciliated cell differentiation. a Heat map of the expression values of genes expressed differentially between E16.5 Shh-Stk11 lungs and control lungs. b Fold change of relative mRNA expression of airway epithelial cell genes in E16.5 Shh-Stk11 lungs compared to Control lungs $(n=3)$. c Fold change of relative mRNA expression of cell-cycle genes in E16.5 Shh-Stk11 lungs compared to Control lungs $(n=3)$. d Pregnant females were oral gavaged daily with a CDK4/6 inhibitor (PD0332991) each day from E12.5 to E14.5. Lungs were analyzed at E16.5. e Cell proliferation was analyzed by antibody staining against Ki67 and E-cadherin (E-cad). $\mathbf{f}_{\text {The }}$ proportion of Ki67 ${ }^{+}$ cells in the intrapulmonary airways of E16.5 lungs (vehicle Control, $n=5$; vehicle Shh-Stk11, $n=3$; PD0332991 Control, $n=7$; PD0332991 Shh-Stk11, $n$ =8). $\mathbf{g}$ Immunofluorescence staining with antibodies against FOXJ1 and SCGB1A1 in lungs after either vehicle or PD0332991 treatment. $\mathbf{h}$ The proportion of FOXJ1 ${ }^{+}$cells (vehicle Control, $n=3$; vehicle Shh-Stk11, $n=4$; PD0332991 Control, $n=3$; PD0332991 Shh-Stk11, $n=5$ ) and the proportion of SCGB1A1 ${ }^{+}$cells $(n=3)$ in the intrapulmonary airways of E16.5 lungs after either vehicle or PD0332991 treatment. i Ten-week-old mice were treated with three doses of tamoxifen. Mice were treated with PD0332991 or vehicle every other day via oral gavage and fed with water containing IdU for 30 days before collecting lungs for analysis. $\mathbf{j}$ Immunofluorescence staining with antibodies against IdU and GFP in lungs after either vehicle or PD0332991 treatment for 30 days. $\mathbf{k}$ The proportion of IdU ${ }^{+}$cells in the GFP labeled intrapulmonary airway epithelium $(n=3)$. I Immunofluorescence staining with antibodies against FOXJ1, GFP, and SCGB1A1 in lungs after either vehicle or PD0332991 treatment for 30 days. The yellow arrows indicate cells expressing both GFP and FOXJ1. $\mathbf{m}$ The proportion of FOXJ1 ${ }^{+} \mathrm{GFP}^{+}$cells to $\mathrm{GFP}^{+}$cells in lungs after either vehicle or PD0332991 treatment for 30 days $(n=3) .{ }^{*} P<0.05 ;{ }^{* *} P<0.01 ;{ }^{* * *} P<0.001$. Data shown in the graphs are means \pm SEM. Student's $t$ test. Scale bars: e, g: $11 \mu \mathrm{m} ; \mathbf{j}, \mathbf{l}: 20 \mu \mathrm{m}$

$\left(\right.$ Ad-STK11 ${ }^{\mathrm{KD}}$ ). The active site lysine residue K78 contributes to both the binding and orientation of ATP, the phosphate donor. Note that the Stk11 ${ }^{\mathrm{K} 78 \mathrm{I}}\left(\mathrm{STK} 11^{\mathrm{KD}}\right)$ variant lacks STK11 kinase activity ${ }^{38}$.

In vitro trachea cultures were established by (i) removing the tracheal rudiments from E13.5 Shh-Stk11 or littermate Control embryos, (ii) making a longitudinal incision to expose the luminal surface and (iii) culturing lumen-side-up at the air-liquid interface on TransWell permeable membrane supports (Fig. 4a). At E13.5, a stage before basal-luminal segregation ${ }^{41}$, no ciliated cells were found in the tracheal epithelium (Supplementary Fig. S4a). Tracheal rudiments were infected with Ad-GFP, AdSTK $11^{\mathrm{WT}}$, or Ad-STK11 ${ }^{\mathrm{KD}}$ viruses and cultured for 5 days (Fig. 4a): we found that the proportions of $\mathrm{Ki} \mathrm{7}^{+}$epithelial cells were significantly decreased in the Shh-Stk11 tracheal rudiments with Ad-STK $11^{\mathrm{WT}}$ infection at postculture day 5 (Fig. 4b, c). No significant change of proliferation was observed on Shh-Stk11 tracheal rudiments with either Ad-GFP or Ad-STK11 ${ }^{\mathrm{KD}}$ infection. Interestingly, when we examined Control tracheal rudiments infected with the Ad-STK $11^{\mathrm{KD}}$ virus, we observed a modest but significant increase in epithelial cell proliferation (Fig. 4b, c), possibly due to a dominant negative effect of the kinase-dead STK11 mutant. Notably, in all of the cultured tracheal rudiments, very few basal cells were observed at the various developmental stages.

We also analyzed ciliated cell formation in cultured tracheal rudiments by performing immunofluorescence staining with an antibody against FOXJ1. The lack of ciliated cell differentiation in Shh-Stk11 tracheal rudiments was rescued by STK11 ${ }^{\mathrm{WT}}$, but not by the kinasedead STK11 ${ }^{\mathrm{KD}}$ (Fig. 4d-g). Consistent with the Ki67 immunostaining results showing a dominant negative effect of STK11 ${ }^{\mathrm{KD}}$ expression in Control tracheal rudiments, the percentage of ciliated cells in the Control tracheal rudiments infected by the Ad-STK $11^{\mathrm{KD}}$ virus decreased significantly compared with those infected by the Ad-GFP virus (Fig. 4d, f, g).

We also conducted experiments in which we reexpressed STK11 within Shh-Stk11 and Control tracheal rudiments. Interestingly, this re-expression of STK11 within Shh-Stk11 tracheal rudiments restored the normal proportion of differentiated ciliated cells without inducing ectopic ciliated cell formation (Fig. 4e, g). In addition, PD0332991 treatment significantly increased the proportion of ciliated cells in the Shh-Stk11 tracheal rudiments but failed to induce ectopic ciliated cell formation in the Control tracheal rudiments (Supplementary Fig. S4b-d). These results indicate that STK11 is necessary but not sufficient for ciliated cell differentiation. Viewed together, these results demonstrate that the kinase activity of STK11 is essential for the function of STK11 in regulating ciliated cell differentiation.

\section{Phosphorylation of MARK3 by STK11 is required for ciliated cell differentiation}

We next investigated downstream phosphorylation targets of STK $11^{39}$. qPCR analysis showed that two of 13 targets are expressed at substantial levels in the airway epithelium of E16.5 lungs: protein kinase AMP-activated catalytic alpha1 subunit (PRKAA1, also known as AMPKo1) and microtubule affinity-regulating kinase 3 (MARK3) (Fig. 5a, Supplementary Fig. S5a, b). Both PRKAA1 and MARK3 are regulated by STK11-mediated phosphorylation $^{39}$. To determine the relative activity of PRKAA1 and MARK3 within developing mouse lung, we performed immunostaining of embryonic lung tissue with phospho-status-specific antibodies. We found that many ciliated cells present in E16.5 lungs had phospho-MARK3 expression (Fig. 5a). Furthermore, the phosphorylation levels of both PRKAA1 and MARK3 were significantly decreased in epithelial cells of Shh-Stk11 embryos compared to the Control, confirming that STK11 is necessary 


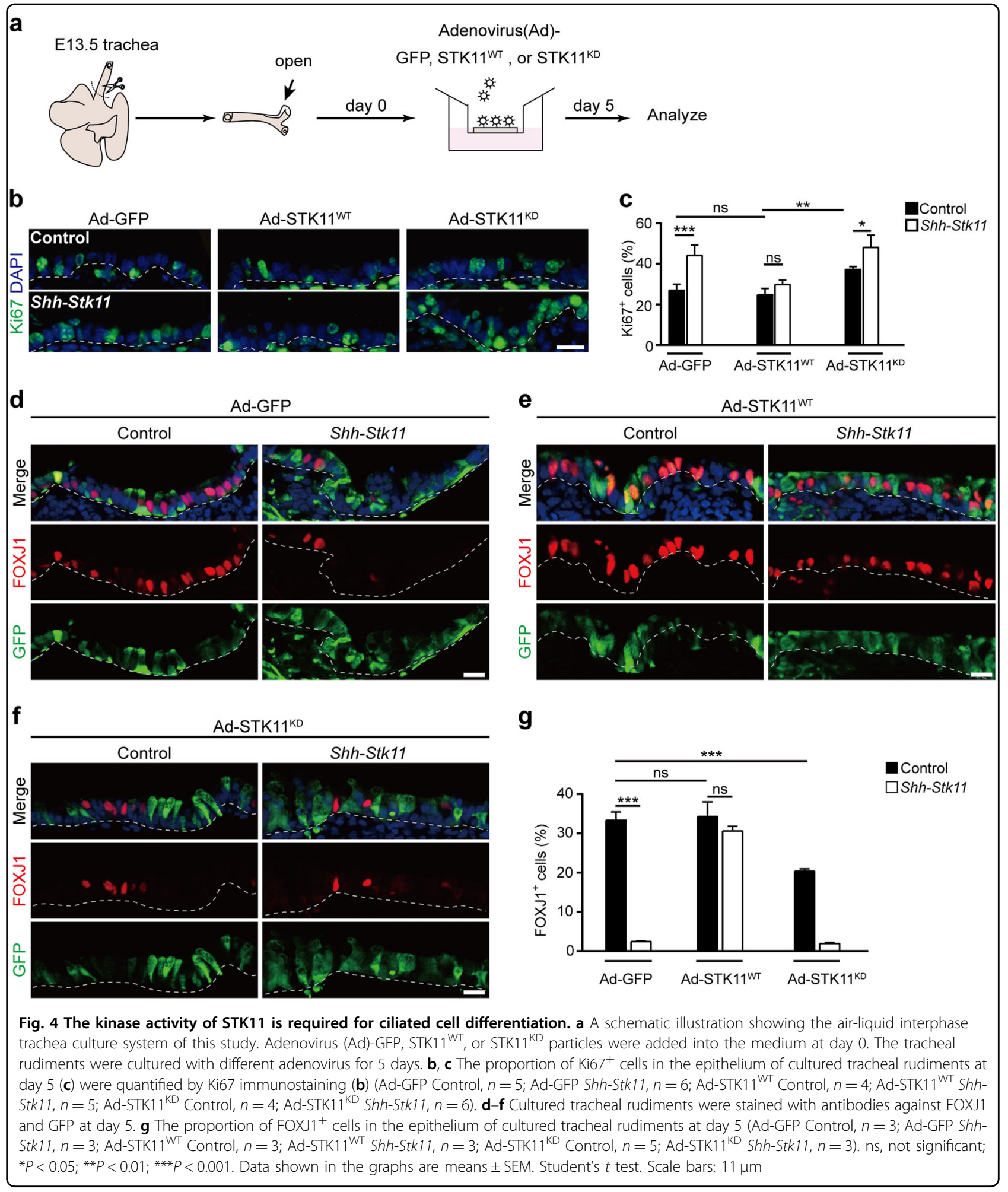

for their activation (Fig. 5a, Supplementary Fig. S5b). We also found, both in vivo and in vitro, that adenosine monophosphate 5-aminoimidazole-4-carboxamide ribonucleotide (AICAR), an activator of PRKAA1 ${ }^{42,43}$, had no impact on either the proliferative status of airway epithelial cells or ciliated cell differentiation (Supplementary Fig. S5c-l); we there therefore subsequently focused on roles for MARK3 as a downstream mediator of STK11. 


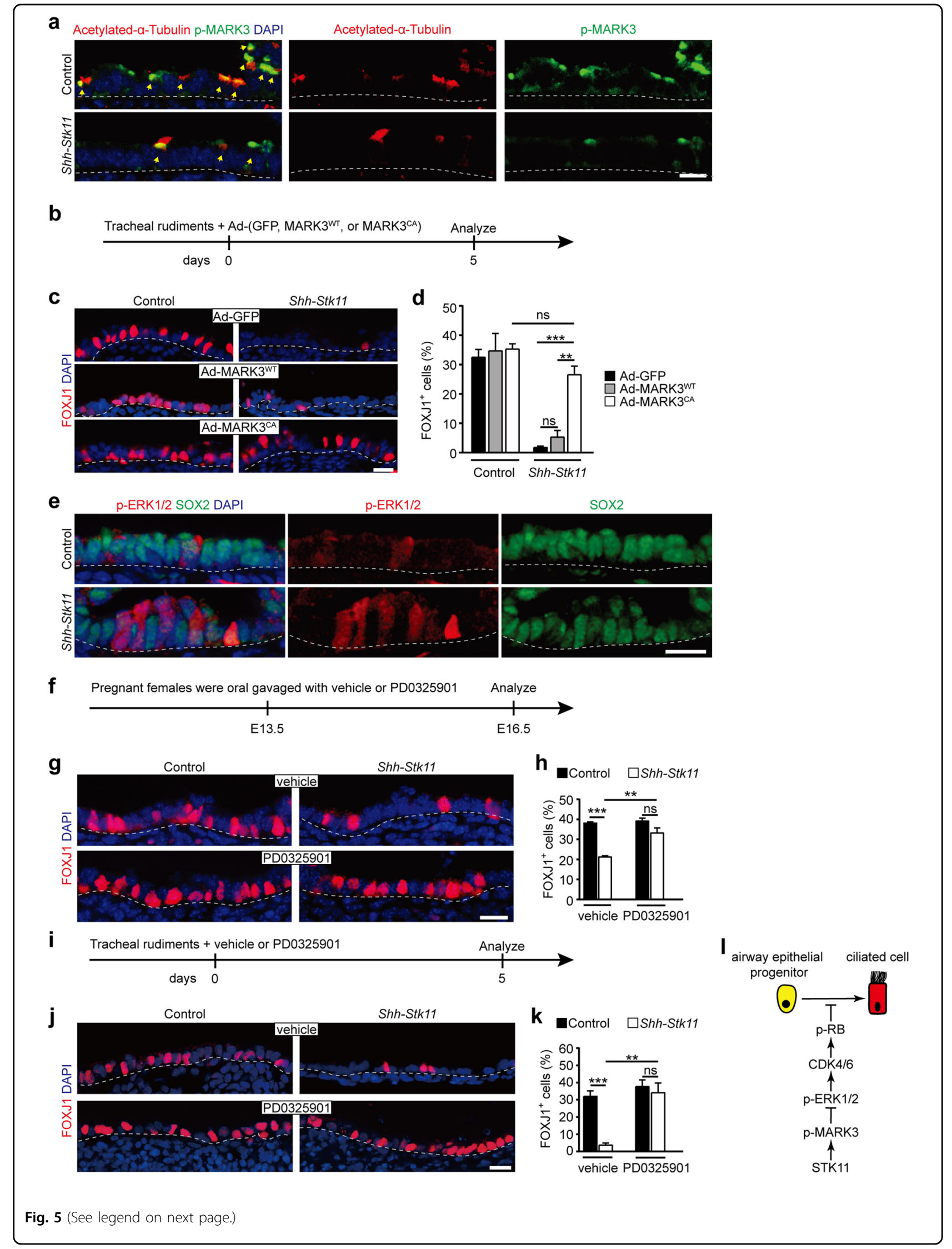


(see figure on previous page)

Fig. 5 A STK11/MARK3 cascade controls ciliated cell differentiation by limiting the activity of ERK1/2 signaling. a Immunofluorescence staining with antibodies against p-MARK3 and acetylated-a-Tubulin in E16.5 lungs. Yellow arrows indicate ciliated cells expressing both acetylated-aTubulin and p-MARK3. b E13.5 tracheal rudiments were cultured with Ad-GFP, Ad-MARK3 ${ }^{W T}$, or Ad-MARK3 ${ }^{\text {CA }}$ viruses for 5 days. c, $\mathbf{d}$ Cultured tracheal rudiments were stained with antibodies against FOXJ1 at day 5 (c). The proportion of FOXJ1 ${ }^{+}$ciliated cells was quantified (Ad-GFP Control, $n=3 ; \mathrm{Ad}-$ GFP Shh-Stk11, $n=3$; Ad-MARK3 ${ }^{\text {WT }}$ Control, $n=3$; Ad-MARK3 ${ }^{\text {WT }}$ Shh-Stk11, $n=4$; Ad-MARK3 ${ }^{\text {CA }}$ Control, $n=4$; Ad-MARK3 $3^{\text {CA }}$ Shh-Stk $\left.11, n=3\right)$ (d). e E16.5 lungs were stained with antibodies against p-ERK1/2 and SOX2. f Pregnant female mice were oral gavaged with p-ERK1/2 inhibitor PD0325901 or vehicle at E13.5. Lungs were analyzed at E16.5. $\mathbf{g}, \mathbf{h}$ Immunofluorescence staining with antibodies against FOXJ1 in the vehicle-treated or PD0325901-treated lungs $(\mathbf{g})$. The proportion of FOXJ1 ${ }^{+}$cells $(n=3)$ and the proportion of SCGB1A $1^{+}$cells $(n=3)$ in the intrapulmonary airways of E16.5 lungs were quantified (h). i E13.5 tracheal rudiments were cultured with vehicle or PD0325901 for 5 days. j, $\mathbf{k}$ Cultured tracheal rudiments were stained with antibodies against FOXJ1 at day 5 (j). The proportion of FOXJ1+ ciliated cells was quantified (vehicle Control, $n=4$; vehicle Shh-Stk $11, n$ $=3$; PD0325901 Control, $n=3$; PD0325901 Shh-Stk11, $n=5)(\mathbf{k})$. I A schematic illustration showing that STK11-mediated signaling cascade accounts for the normal program of ciliated cell differentiation in airways. ns, not significant; ${ }^{*} P<0.05 ;{ }^{* *} P<0.01 ;{ }^{* *} P<0.001$. Data shown in the graphs are means \pm SEM. Student's $t$ test. Scale bars: $11 \mu \mathrm{m}$

We used recombinant adenoviral vectors expressing a Flag-tagged constitutively activated MARK3 mutant (AdMARK $3^{\text {CA }}$, the substitution of threonine 211 by a glutamic acid) to examine its role as a downstream target of STK11 that may mediates its effects on ciliated cell differentiation. Tracheal rudiment cultures prepared from E13.5 Shh-Stk11 or Control embryos were infected with Ad-GFP, Ad-MARK3 ${ }^{\mathrm{WT}}$, or Ad-MARK $3^{\mathrm{CA}}$ viruses, followed by culturing for 5 days (Fig. 5b, Supplementary Fig. S6a). Infection of Shh-Stk11 tracheal rudiments with the Ad-MARK $3^{\mathrm{CA}}$ virus led to a significant reduction in the proportion of $\mathrm{Ki} 7^{+}$epithelial cells compared to tracheal rudiments infected with either the Ad-GFP or AdMARK3 ${ }^{\text {WT }}$ virus (Supplementary Fig. S6b, c). These results show that the proliferative defect observed in rudiments with STK11 deletion can be rescued by the activation of MARK3; such activation actually reduced the proliferative index of the $S h h-S t k 11$ epithelium to the level seen in the Control epithelium.

Examination of the ciliated cells in these virus-infected tracheal rudiments further supported a direct role for activated MARK3 in ciliated cell differentiation. Specifically, infection of Shh-Stk11 tracheal rudiments with the Ad-MARK ${ }^{\text {CA }}$ virus significantly increased the percentage of ciliated cells as compared to Shh-Stk11 tracheal rudiments infected with the Ad-GFP or Ad-MARK3 ${ }^{\text {WT }}$ viruses (Fig. 5c, d). Thus, our support that STK11-dependent phosphorylation of MARK3 is required for cell-cycle exit and ciliated cell differentiation during airway epithelial development.

\section{A STK11/MARK3 signaling cascade controls ciliated cell differentiation by limiting ERK1/2 signaling activity}

Given the known function of MARK3 as an inhibitor of ERK1/2 signaling ${ }^{44,45}$-a pro-mitotic signaling pathway ${ }^{46,47}$ - we next determined whether the effects of $\mathrm{p}$ MARK3 on cell-cycle progression and ciliated cell differentiation within airway epithelial progenitor cells are mediated through inhibition of ERK1/2. To test this, we initially analyzed the presence of p-ERK $1 / 2$ in $S O X 2^{+}$ intrapulmonary airway epithelial cells of E16.5 Control and Shh-Stk11 lungs. SOX2 ${ }^{+}$epithelial cells of Shh-Stk11 lungs showed a significant increase in p-ERK1/2 immunoreactivity compared to epithelial cells of Control lungs (Fig. 5e). Moreover, the increased expression level of pERK1/2 was observed in cultured Shh-Stk11 tracheal rudiments, and expression of MARK ${ }^{\mathrm{CA}}$ in cultured ShhStk11 tracheal rudiments significantly decreased the number of epithelial cells showing p-ERK1/2 immunoreactivity (Supplementary Fig. S6d), findings that support a mechanism wherein p-MARK3 controls cell-cycle exit via inhibition of ERK $1 / 2$ signaling.

To examine the role of ERK1/2 signaling in ciliated cell differentiation, we treated pregnant females with a specific MEK1/2 inhibitor (PD0325901) or vehicle control at E13.5 and analyzed lungs at E16.5 (Fig. 5f). The number of cells expressing p-ERK1/2 in PD0325901-treated ShhStk11 lungs was significantly decreased compared with vehicle-treated Shh-Stk11 lungs (Supplementary Fig. S6e). Both quantification of $\mathrm{Ki}^{+} 7^{+}$intrapulmonary airway epithelial cells and PI staining showed that the proportion of proliferating epithelial cells in Shh-Stk11 lungs was significantly reduced by the PD0325901 treatment (Supplementary Fig. S6f, g). Importantly, these experiments also showed that PD0325901 treatment rescued the impaired ciliated cell differentiation in the lungs of Shh-Stk11 embryos in vivo (Fig. 5g, h) and in the cultured Shh-Stk11 tracheal rudiments in vitro (Fig. $5 \mathrm{i}-\mathrm{k}$ ). Moreover, these results show that increased ERK1/2 signaling in Shh-Stk11 lungs promotes cell-cycle progression and interferes with the differentiation of ciliated cells. Thus, our findings define a STK11/MARK3/ERK1/2 signaling cascade allows a normal differentiation program of ciliated cells (Fig. 5l).

\section{A STK11/MARK3/ERK1/2 signaling cascade functions to enforce ciliated cell lineage commitment}

Considering our results demonstrating that the requirement of STK11/MARK3/ERK1/2 signaling cascade 
in regulating ciliated cell differentiation, we were interested in understanding the relationship between STK11 and ciliated cell fate specification. It is firmly established that Notch signaling determines the fate of lung airway epithelial cells by promoting secretory over ciliated cell fates $^{4,6,8,10}$. We therefore compared the mRNA expression levels of multiple Notch ligands, receptors, and downstream targets in both Control and Shh-Stk11 lungs of E13.5 embryos, a stage at which airway epithelial progenitor cells have not committed to either secretory or ciliated cell fates (Supplementary Fig. S7a). The similar expression levels of Notch pathway genes (Notch1, Notch2, Jag1, Hes1, and Hey1) that we observed in the lungs of Shh-Stk11 embryos clearly implied that STK11 does not directly regulate the expression of canonical Notch pathway genes.

To further investigate the possible relationship(s) between STK11 and Notch signaling, we generated Shh-

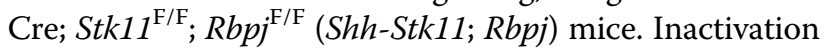
of Notch the signaling pathway by deleting Rbpj, a DNAbinding protein that associates with Notch intracellular domain to transcriptionally regulate downstream genes expression $^{48}$, promotes ciliated cell development and when Notch signaling is absent almost all airway epithelial progenitor cells differentiate into ciliated cells ${ }^{4,6,8}$. We first analyzed the cell proliferation in lungs of E13.5 embryos and found the proportions of $\mathrm{PH}^{+}$airway epithelial cells were not significantly different between Control and Shh-Cre; $\operatorname{Rbpj}^{\mathrm{F} / \mathrm{F}}$ (Shh-Rbpj) lungs. The increased proportions of $\mathrm{PH}^{+}$epithelial cells were not significantly different between the Shh-Stk11 and the double mutant lungs (Supplementary Fig. S7b, c). This result is consistent with a previous study which found that the loss of Rbpj does not influence cell proliferation in the adult airway epithelium ${ }^{10}$. Additionally, this finding further confirms our conclusion that STK11-regulated cellcycle exit functions independently of Notch signaling activity.

Having established the independence of STK11regulated cell-cycle exit and Notch signaling, we were interested to determine whether these pathways exert their effects on ciliated cell differentiation simultaneously. We therefore assessed the proportions of club cells versus ciliated cells in the lungs of the aforementioned four genotypes by immunostaining using antibodies against SCGB1A1 and FOXJ1. In E16.5 lungs of Shh-Rbpj mice, $90.56 \pm 0.34 \%$ intrapulmonary airway epithelial cells were FOXJ1 $1^{+}$ciliated cells, and $5.34 \pm 0.13 \%$ of the intrapulmonary airway epithelial cells expressed neither SCGB1A1 nor FOXJ1; we did not find detectable apoptosis in these lungs (Supplementary Figs. S3h and 7d). Interestingly, the proportion of FOXJ1 $1^{+}$ciliated cells was significantly decreased in E16.5 Shh-Stk11; Rbpj lungs $(52.23 \pm 1.28 \%)$, and the proportion of SCGB1A1-FOXJ1- intrapulmonary airway epithelial cells increased to $43.4 \pm$ $1.89 \%$ in E16.5 Shh-Stk11; Rbpj lungs (Fig. 6a, b). However, by E18.5, the proportion of FOXJ1 $1^{+}$ciliated cells in Shh-Stk11; Rbpj lungs increased to $90.83 \pm 1.92 \%$ (Fig. 6c, d). In addition, we found that many of these SCGB1A1 ${ }^{-}$FOXJ1 $^{-}$cells were positive for Ki67 in E16.5 Shh-Stk11; Rbpj lungs (Supplementary Fig. S7e, f). Therefore, these results indicate that the SCGB1A1-. FOXJ1- $1^{-}$cells in E16.5 Shh-Stk11; Rbpj lungs ultimately differentiated into $\mathrm{FOXJ1}^{+}$ciliated cells at E18.5, suggesting that these SCGB1A1 ${ }^{-}$FOXJ1 $1^{-}$cells have committed to an early ciliated cell fate (pre-ciliated cells). Thus, as the lack of Notch signaling strongly promoted differentiation of airway epithelial cells toward a ciliated cell fate in Shh-Stk11; Rbpj lungs, we can conclude that the regulatory influence of STK11/MARK3/ERK1/2 signaling cascade on ciliated cell differentiation is not related to ciliated cell fate specification.

JAGGED1 (JAG1) is a Notch ligand that is known to be expressed by ciliated cells in the intrapulmonary airways ${ }^{8}$. To investigate the expression level of Jagged1, we isolated $\mathrm{EpCAM}^{+} \mathrm{CD}_{4}{ }^{-} \mathrm{CD} 31^{-}$epithelial cells from Control lungs and Shh-Stk11 lungs at E14.5, E16.5, and E18.5. Surprisingly, we found that the mRNA level of Jagged1 was not altered in Shh-Stk11 lungs at E14.5 and E16.5 (Supplementary Fig. S7g). In light of our previous finding that the proportion of ciliated cells decreased in E16.5 Shh-Stk11 lungs (Fig. 2a, b), we also performed immunostaining using antibodies against JAGGED1 and acetylated- $\alpha$-tubulin. We found that the proportion of JAGGED1 $^{+}$cells was not decreased and that many acetylated- $\alpha$-tubulin negative cells expressed JAGGED1 in the E16.5 Shh-Stk11 lungs (Fig. 6e, f). These results suggest that the acetylated- $\alpha$-tubulin ${ }^{-}$JAGGED1 ${ }^{+}$cells may have committed to ciliated cell fates but cannot perform multiciliogenesis to terminally differentiate into acetylated- $\alpha$-tubulin ${ }^{+}$ciliated cells in the Shh-Stk11 lungs. At E18.5, both the mRNA level of Jagged1 and the proportion of JAGGED1 ${ }^{+}$cells were decreased in the Shh-Stk11 lungs, likely due to the significantly reduced proportion of ciliated cells at this stage (Fig. 6g, h, Supplementary Fig. S7g). Taken together, our findings indicate that STK11 acts to ensure ciliated cell fate commitment.

\section{Discussion}

In our study, we identified that STK11-in both embryonic and adult lungs-is required for the normal program of ciliated cell differentiation. In the absence of Stk11, ciliated cell differentiation is largely impaired. When we inhibited the phosphorylation level of $\mathrm{pRB}$ by the PD0332991 treatment, both the increased cell proliferation and impaired ciliated cell differentiation were restored in Shh-Stk11 lungs. Furthermore, we demonstrated that STK11 regulates cell-cycle exit and ciliated 


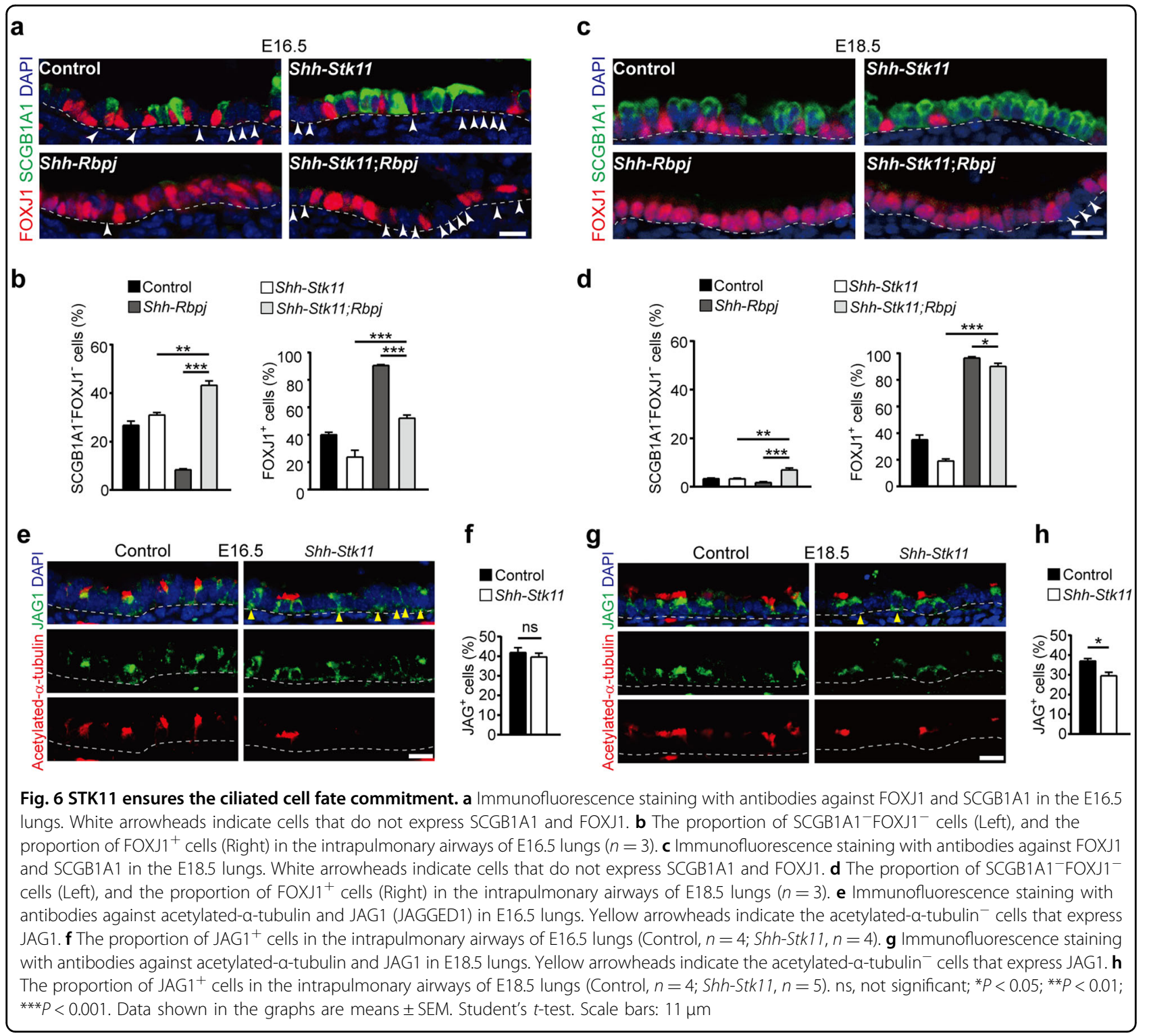

cell differentiation by phosphorylating its downstream target, MARK3, which in turn leads to the inhibition of ERK1/2 signaling-mediated pRB phosphorylation ${ }^{37,44,49}$. Given the clear evidence that decreased expression of STK11 in human diseases is associated with ciliopathies, we conclude that STK11 is a physiological regulator to enforce ciliated cell fate commitment and multiciliogenesis, ensuring the normal program of ciliated cell differentiation.

Notch signaling controls the balance of ciliated and secretory cell fates in developing airways ${ }^{8}$. We found no evidence to support a direct interaction between the STK11 and Notch signaling pathways. It is established that inactivated Notch signaling induces neuroendocrine cell fate specification ${ }^{50}$. The fact that neuroendocrine cell differentiation is not significantly different between Shh-
Stk11 and Control lungs provides further evidence that Notch signaling is not impaired in Shh-Stk11 lungs (Supplementary Fig. S2k, l). The relative expression level of Jagged1 is not significantly decreased in the E13.5, E14.5, or E16.5 Shh-Stk11 lungs, and the expression of JAGGED1 in many acetylated- $\alpha$-tubulin ${ }^{-}$cells of E16.5 Shh-Stk11 lungs suggest that these airway epithelial progenitors may have acquired the ciliated cell fate.

Once they have adopted a low Notch signalingdependent ciliated cell fate, the preciliated cells initiate the expression of ciliary genes to launch the multiciliogenesis process. It has been demonstrated that multiciliogenesis is a precise process sequentially regulated by the nucleocytoplasmic translocation of $\mathrm{E} 2 \mathrm{~F} 4{ }^{13}$. Once the ciliated cell fate is determined, nuclear E2F4-DP1Multicilin complex initiates a transcriptional program of 
centriole biogenesis and turns on genes that are required for multiciliogenesis (e.g., Deup1, Myb, Ccno, and Foxj 1$)^{15,21,51-54}$. Then, E2F4 translocates to cytoplasm and organizes deuterosome assembly centers to initiate centriole amplification ${ }^{13}$. In our study, we found that both the transcriptional coactivator Multicilin (Mcidas) and genes that are transcriptionally regulated by E2F4-DP1Multicilin complex (Deup1, Myb, Ccno, and Foxj1), were significantly downregulated in Shh-Stk11 lungs (Fig. 3a, b, Supplementary Dataset S1). This finding demonstrates that loss of Stk11 impairs the transcriptional program of centriole biogenesis. Furthermore, the increased proportion of club cells observed in Shh-Stk11 lungs suggests that diminished expression of centriole biogenesis genes may causes loss of ciliated cell identity in pre-ciliated cells, allowing these cells to assume a non-ciliated cell fate. We therefore propose that STK11/MARK3/ERK1/2 signaling cascade is required for preventing the plasticity of preciliated cells and ensuring their commitment to multiciliogenesis.

Multiciliated cells are the main constituents of mucociliary epithelia, fulfilling critical roles in epithelia of respiratory, nervous, and reproductive systems by providing the mechanical force to mobilize fluids and external particles across the epithelium. These functions rely on proper ciliated cell differentiation during both development and homeostasis. Understanding the molecular mechanisms underlying the step-by-step ciliated cell differentiation process will provide insight for our future understanding of ciliated cell-related diseases.

\section{Materials and methods}

\section{Mice}

Shh-Cre ${ }^{55}$, Sox2-CreER ${ }^{55}$, Rosa26-mTmG ${ }^{56}$, Scgb1a1$\mathrm{CreER}^{24}$, floxed $R b p j^{57}$, and floxed $S t k 11^{58}$ mice have been described previously. Mice had mixed genetic backgrounds. Noon of the day on which a vaginal plug was detected was considered E0.5. Tamoxifen $(75 \mu \mathrm{g} / \mathrm{g}$ body weight) or corn oil was injected into Scgb1a1-CreER, floxed Stk11, Rosa26-mTmG mice, and mice carrying Sox2-CreER, floxed Stk11 and their respective control embryos. Mice were killed using $\mathrm{CO}_{2}$. All experiments were performed in accordance with the recommendations in the Guide for Care and Use of Laboratory Animals of the National Institute of Biological Sciences, Beijing and Cedars-Sinai Medical Center, Los Angeles. All experiments were approved by the Institutional Review Board at the National Institute of Biological Science, Beijing and Cedars-Sinai Medical Center, Los Angeles.

\section{scRNA-seq analysis}

$\mathrm{CD} 45^{-} \mathrm{CD}^{-} 1^{-} \mathrm{EpCAM}^{+}$lung epithelial cells from embryonic lungs at E16.5 and adult lung of four male and four female mice (two batches) were FACS sorted and performed for scRNA-seq analysis using the $10 \times$ Genomic Chromium system, respectively. CellRanger v2.2.0 software was used with the default settings for demultiplexing, aligning reads with STAR software to $\mathrm{mm}$ 10 mouse reference genome, and counting unique molecular identifiers. Downstream analysis was performed using Seurat v2.3R package. Uniform Manifold Approximation and Projection (UMAP) method has been used for visualization of unsupervised clustering. The cell type of each cluster is determined by known markers of individual cell types. Airway cells are further subset for expression and pseudo-time analysis. Pseudo-time trajectories are performed using Monocle 2R package ${ }^{59}$.

\section{FACS and cell-cycle analysis}

The E16.5 lungs were cut into pieces smaller than $1 \mathrm{~mm}^{3}$ after removing the heart. Tissues were then digested using a neutral protease (Worthington Biochemical Corporation) and DNase I (Roche) at $37^{\circ} \mathrm{C}$ for $20 \mathrm{~min}$. The reaction was stopped using DMEM medium supplemented with 10\% FBS. The tissue suspension was passed through a $40 \mu \mathrm{m}$ mesh filter (Thermo Scientific) to remove undigested tissue fragments. The supernatant was centrifuged at $300 \mathrm{~g}$ for $10 \mathrm{~min}$ and resuspended with red blood lysis buffer. After centrifuging again, the cells were resuspended with blocking buffer (5\% BSA in DPBS) and stained with PE-Cy7 rat anti-CD31 (1:400, 561410, BD biosciences), APC mouse anti-EpCAM (1:400, 17-579182, eBioscience), and FITC rat anti-CD45 (1:400, 553080, BD biosciences) for $20 \mathrm{~min}$ on ice. DAPI was used to exclude dead cells before sorting. The $\mathrm{EpCAM}^{+} \mathrm{CD} 45^{-} \mathrm{CD} 31^{-}$cells were then sorted via flow cytometry (Aria II, BD Biosciences). The isolated cells were centrifuged at $300 \mathrm{~g}$ for $10 \mathrm{~min}$. Then they were washed with cold DPBS twice. The cells were resuspended and fixed with precold $70 \%$ ethanol for $24 \mathrm{~h}$ at $4{ }^{\circ} \mathrm{C}$. For cell-cycle analysis, the fixed cells were stained with PI (P4170, Sigma) supplemented with Rnase A (CW0600S, cwbiotech Corporation) for $15 \mathrm{~min}$ at RT. The cell-cycle status was also analyzed by flow cytometry and the data were processed using Flowjo v10 (BD biosciences).

\section{Administration of inhibitors to mice}

The pregnant females were treated with PD0332991 $(150 \mathrm{mg} / \mathrm{kg}$, Sigma) by oral gavage from E12.5 to E14.5 each day or with PD0325901 (5 mg/kg, Sigma) by oral gavage at E13.5 or with AICAR $(250 \mathrm{mg} / \mathrm{kg}$, Sigma) by intraperitoneal injection from E12.5 to E14.5 each day. Ten-week-old mice were treated with PD0332991 (50 mg/ $\mathrm{kg}$, Sigma) every other day for 30 days by oral gavage.

\section{Trachea culture}

The tracheal rudiments were dissected from E13.5 lungs. These tracheal rudiments were made a longitudinal 
incision with a sharp needle on ice as quickly as possible. The tracheal rudiments were then washed three times in HBSS (Gibco, 14025-092) supplemented with penicillin/ streptomycin for $5 \mathrm{~min}$ on ice. We next transferred these tracheal rudiments to TransWell units and cultured them at an air-liquid interface of DMEM/F12 medium (Gibco, 11039-021). The tracheal rudiments were cultured for 5 days in vitro. The day when the tracheal rudiments were dissected and cultured in the medium was defined as day 0 . For adenovirus infection experiment, the adenovirus was added into the medium at day 0 . The virus titer finally used was $10^{8}(\mathrm{PFU} / \mathrm{ml})$. The tracheal rudiments were cultured with adenovirus for $24 \mathrm{~h}$. For the inhibitor chemical treatment experiments, AICAR $(10 \mu \mathrm{M})$, PD0325901 $(10 \mu \mathrm{M})$, or PD0332991 $(5 \mu \mathrm{M})$ was added to the medium at day 0 and withdraw after $72 \mathrm{~h}$ treatment, respectively. The medium was changed every day in all experiments.

\section{RNA sequencing and data processing}

Total RNA was extracted from E16.5 lungs using a Direct-zol $^{\mathrm{TM}}$ RNA MiniPrep kit (ZYMO RESEARCH, R2050). The sequencing was performed at the Sequencing Center of NIBS with SE50 using a Hiseq-2500 instrument. RNA-seq analyses of E16.5 lungs were performed with at least two independent biological replicates. Enriched GO and KEGG pathway terms for RNA-seq differentially expressed gene sets were identified using DAVID. Visualization of RNA-seq was performed using the Integrative Genomics Viewer.

\section{Immunohistochemistry}

Mouse lungs were fixed in $4 \%$ paraformaldehyde at $4{ }^{\circ} \mathrm{C}$ overnight. For frozen sections, lungs were immersed in sucrose, and then embedded in OCT. For paraffin sections, lungs were dehydrated in a gradient of ethanol, made transparent with xylene, and embedded in wax. Immunofluorescence staining was performed using the following primary antibodies: rabbit anti-STK11 antibody (Cell Signaling, 13031, 1:500), mouse anti-acetylated- $\alpha$ Tubulin (Sigma, T6793, 1:2000), mouse anti-FOXJ1 (eBioscience, 14-9965, 1:500), mouse anti-MYB (Santa Cruz, sc-74512, 1:500), rabbit anti-Ki67 (Abcam, ab15580, 1:300), chicken anti-GFP antibody (Abcam, ab13970, 1:1000), rabbit anti-AMPKa (PRKAA1) antibody (Cell Signaling, $2535 \mathrm{~s}, 1: 500$ ), rat anti-E-Cadherin (Invitrogen clone ECCD-2, 1:500), rabbit anti-PH3 (Millipore, 06-570, 1:100), rabbit anti-SCGB1A1 (Millipore, 07-623, 1:300), rabbit anti-phosphorylated MARKs antibody (Cell Signaling, 4836, 1:200), rabbit anti-Caspase3 antibody (Cell Signaling, 9664s, 1:300), goat anti-SOX2 antibody (Santa Cruz, sc-17320, 1:100), mouse anti-P63 (Santa Cruz, sc-8431, 1:500), rabbit anti-CGRP antibody (Sigma, C8198, 1:300), mouse anti-Flag antibody (Sigma, A2220,
1:200), rabbit anti-phosphorylated ERK1/2 antibody (Cell Signaling, 4370S, 1:200), rabbit anti-p-pRB antibody (Cell Signaling, 8516, 1:1600), rabbit anti-JAG1 antibody (Cell Signaling, 70109, 1:1000), and mouse anti-IdU antibody (BD Biosciences, 347580, 1:500). The following secondary antibodies were used: Alexa Fluor 488-Donkey anti-goat (Jackson Immuno Research), Alexa Fluor Cy3-Donkey anti-rabbit (Jackson Immuno Research), Alexa Fluor 488Donkey anti-chicken (Jackson Immuno Research), Alexa Fluor 568-Donkey anti-rat (Jackson Immuno Research), and Alexa Fluor 647-Donkey anti-rat (Jackson Immuno Research), Alexa Fluor 647-Donkey anti-rabbit (ThermoFisher Scientific), Alex Fluor 568-Donkey anti-mouse IgG $(\mathrm{H}+\mathrm{L})$ (ThermoFisher Scientific). All of the Alexa Fluor coupled secondary antibodies were used at 1:500 dilutions.

Immunohistochemical staining for STK11, phosphorylated MARKs, MYB, and FOXJ1 were performed using a TSA Biotin Tyramide Amplification system (SAT700001EA, Perkin Elmer) for amplification. The following secondary antibodies were used: Biotin-SPconjugated AffiniPure Donkey anti-rabbit (Jackson Immuno Research), Biotinylated horse anti-mouse (Vector Laboratories). The cultured tracheas were collected and fixed in $4 \%$ paraformaldehyde at $4{ }^{\circ} \mathrm{C}$ for $1 \mathrm{~h}$. Then they were immersed in sucrose and embedded in OCT.

\section{Microscopy and imaging}

Tissue section immunofluorescence staining was imaged using confocal microscopy (Leica Corporation). Cells were counted based on the basis of nuclear staining with DAPI and specific cell markers of the respective cell types. Cells were counted using $\times 40$ magnification fields covering the whole left lung. Images were processed and analyzed using Image (NIH), Adobe Photoshop (Adobe), and Adobe Illustrator (Adobe).

\section{Adenovirus production}

The genes encoding the kinase-dead variants of Stk11 and wild-type Stk11 were cloned from the plasmids kindly provided by Dr Hongbin Ji. To disable the kinase activity of STK11, the lysine residue at position 78 was mutated into isoleucine. The Mark3 ${ }^{C A}$ gene was synthesized by Obio Technology (Shanghai). The STK11 WT adenovirus and STK $11^{\mathrm{KD}}$ adenovirus were constructed by cloning wild-type Stk11 and kinase-dead variants of Stk11 into the vector pAdeno-MCMV-EGFP-P2A-MSC3FLAG. The MARK $3^{\mathrm{CA}}$ and MARK $3^{\mathrm{WT}}$ adenovirus were constructed by cloning the mutant MARK3 and wild type MARK3 into the vector pAdeno-MCMV-3FLAG-P2AEGFP. The GFP-adenovirus, STK11 ${ }^{\mathrm{WT}}$ adenovirus, STK $11^{\mathrm{KD}}$ adenovirus, MARK $3^{\mathrm{WT}}$ adenovirus, and MARK $3^{\mathrm{CA}}$ adenovirus were purchased from Obio Technology (Shanghai). 


\section{Analyze the adenovirus infection efficacy}

The infection efficacy of Ad-GFP, Ad-STK11 ${ }^{\mathrm{WT}}$, and Ad-STK $11^{\mathrm{KD}}$ was determined by performing immunofluorescence staining with an antibody against GFP. The infection efficacy of Flag-tagged Ad-GFP, Ad-MARK $3^{\text {WT }}$ and Ad-MARK3 ${ }^{\mathrm{CA}}$ was determined by performing immunofluorescence staining with an antibody against Flag.

\section{RNA extraction and qPCR}

The total RNA of E16.5 lungs was extracted using Trizol reagent (Ambion) and Direct-Zol RNA Miniprep kits (ZYMO research). RNA was reverse transcribed into firststrand complementary DNA using HiScript II Q RT SuperMix (Vazyme). The qPCR analysis was performed on a CFX96 Tough instrument (Bio-Rad) using KAPA SYBR FAST qPCR Master Mix (KAPA). The primers were provided in Supplementary Table S1.

\section{Statistical analysis}

All in vivo quantifications are based on the airway epithelial cells of the intrapulmonary airways in embryonic lungs. All quantification data are presented as the mean \pm SEM. (as indicated in figure legends). All quantification data presented in the figures were collected from at least three experimental replications per group on different days using different mice. We used two-tailed Student's $t$ tests to assess differences between means.

\section{Acknowledgements}

We thank Dr Hongbin Ji for providing us the plasmids of STK $11^{\mathrm{WT}}$ and STK $11^{\mathrm{KD}}$. We thank for Dr Xiangrong Guan for technique support. This work was funded by the National Institutes of Health 1T32HL134637 and R01HL135163; California Institution of Regenerative Medicine CIRM LA1-06915. This work was funded by Beijing Major Science and Technology Projects, Z171100000417003.

\section{Author details}

${ }^{1}$ College of Life Sciences, Beijing Normal University, 100875 Beijing, China. ${ }^{2}$ National Institute of Biological Sciences, 102206 Beijing, China. ${ }^{3}$ Lung and Board of Governors Regenerative Medicine Institutes, Department of Medicine, Cedars-Sinai Medical Center, 90048 Los Angeles, CA, USA. ${ }^{4}$ Tsinghua Institute of Multidisciplinary Biomedical Research, Tsinghua University, 100084 Beijing, China

\section{Authors' contributions}

Q.C. and C.Y. handled the following: study design, characterization of animal model, immunohistochemistry data analysis and interpretation, and writing of manuscript. X.Q. helped with the inhibitor experiment. B.R.S. and N.T. codesigned the experiments and co-wrote the manuscript.

\section{Data availability}

The RNA-seq data along with their associated meta data have been deposited in the GEO database under accession code GSE116690 and GSE123838. All relevant data of this study are available from the authors upon reasonable request.

\section{Conflict of interest}

The authors declare that they have no conflict of interest.

\section{Publisher's note}

Springer Nature remains neutral with regard to jurisdictional claims in published maps and institutional affiliations.

Supplementary Information accompanies the paper at (https://doi.org/ 10.1038/s41421-019-0104-z).

Received: 27 March 2019 Revised: 6 May 2019 Accepted: 31 May 2019 Published online: 16 July 2019

\section{References}

1. Rackley, C. R. \& Stripp, B. R. Building and maintaining the epithelium of the lung. J. Clin. Investig. 122, 2724-2730 (2012).

2. Hogan, B. L. et al. Repair and regeneration of the respiratory system: complexity, plasticity, and mechanisms of lung stem cell function. Cell Stem Cell 15, 123-138 (2014).

3. Morimoto, M. et al. Canonical Notch signaling in the developing lung is required for determination of arterial smooth muscle cells and selection of Clara versus ciliated cell fate. J. Cell Sci. 123, 213-224 (2010).

4. Morimoto, M., Nishinakamura, R., Saga, Y. \& Kopan, R. Different assemblies of Notch receptors coordinate the distribution of the major bronchial Clara, ciliated and neuroendocrine cells. Development 139, 4365-4373 (2012).

5. Morrisey, E. E. \& Hogan, B. L. Preparing for the first breath: genetic and cellular mechanisms in lung development. Dev. Cell 18, 8-23 (2010).

6. Rock, J. R. et al. Notch-dependent differentiation of adult airway basal stem cells. Cell Stem Cell 8, 639-648 (2011).

7. Tsao, P. N. et al. Gamma-secretase activation of notch signaling regulates the balance of proximal and distal fates in progenitor cells of the developing lung. J. Biol. Chem. 283, 29532-29544 (2008).

8. Tsao, P. N. et al. Notch signaling controls the balance of ciliated and secretory cell fates in developing airways. Development 136, 2297-2307 (2009).

9. Tsao, P. N. et al. Notch signaling prevents mucous metaplasia in mouse conducting airways during postnatal development. Development 138, 3533-3543 (2011).

10. Pardo-Saganta, A. et al. Parent stem cells can serve as niches for their daughter cells. Nature 523, 597-601 (2015).

11. Tadokoro, $T$. et al. IL-6/STAT3 promotes regeneration of airway ciliated cells from basal stem cells. Proc. Natl Acad. Sci. USA 111, E3641-E3649 (2014).

12. McConnell, A. M. et al. p53 regulates progenitor cell quiescence and differentiation in the airway. Cell Rep. 17, 2173-2182 (2016).

13. Mori, M. et al. Cytoplasmic E2f4 forms organizing centres for initiation of centriole amplification during multiciliogenesis. Nat. Commun. 8, 15857 (2017).

14. Stubbs, J. L., Vladar, E. K., Axelrod, J. D. \& Kintner, C. Multicilin promotes centriole assembly and ciliogenesis during multiciliate cell differentiation. Nat. Cell Biol. 14, 140-147 (2012).

15. Gomperts, B. N., Gong-Cooper, X. \& Hackett, B. P. Foxj1 regulates basal body anchoring to the cytoskeleton of ciliated pulmonary epithelial cells. J. Cell Sci. 117, 1329-1337 (2004).

16. Gascue, C. et al. Direct role of Bardet-Biedl syndrome proteins in transcriptional regulation. J. Cell Sci. 125, 362-375 (2012).

17. Flowers, E. M. et al. Lkb1 deficiency confers glutamine dependency in polycystic kidney disease. Nat. Commun. 9, 814 (2018).

18. Stokman, M. F. et al. Changes in the urinary extracellular vesicle proteome are associated with nephronophthisis-related ciliopathies. J. Proteom 192, 27-36 (2019).

19. Chen, $H$. et al. Airway epithelial progenitors are region specific and show differential responses to bleomycin-induced lung injury. Stem Cells 30, 1948-1960 (2012).

20. Sanchez-Cespedes, M. et al. Inactivation of LKB1/STK11 is a common event in adenocarcinomas of the lung. Cancer Res. 62, 3659-3662 (2002).

21. Tan, F. E. et al. Myb promotes centriole amplification and later steps of the multiciliogenesis program. Development 140,4277-4286 (2013).

22. Que, J. et al. Multiple dose-dependent roles for Sox2 in the patterning and differentiation of anterior foregut endoderm. Development 134, 2521-2531 (2007).

23. Evans, C. M. et al. Mucin is produced by clara cells in the proximal airways of antigen-challenged mice. Am. J. Respir. Cell Mol. Biol. 31, 382-394 (2004) 
24. Rawlins, E. L. et al. The role of Scgbla1+ Clara cells in the long-term maintenance and repair of lung airway, but not alveolar, epithelium. Cell Stem Cell 4, 525-534 (2009).

25. Treutlein, B. et al. Reconstructing lineage hierarchies of the distal lung epithelium using single-cell RNA-seq. Nature 509, 371-375 (2014).

26. Weis, K. Regulating access to the genome: nucleocytoplasmic transport throughout the cell cycle. Cell 112, 441-451 (2003).

27. Branzei, D. \& Foiani, M. Regulation of DNA repair throughout the cell cycle. Nat. Rev. Mol. Cell Biol. 9, 297-308 (2008).

28. Scholzen, T. \& Gerdes, J. The Ki-67 protein: from the known and the unknown. J. Cell. Physiol. 182, 311-322 (2000).

29. Otto, T. et al. Cell cycle-targeting microRNAs promote differentiation by enforcing cell-cycle exit. Proc. Natl Acad. Sci. USA 114, 10660-10665 (2017).

30. Bettencourt-Dias, M. et al. Genome-wide survey of protein kinases required for cell cycle progression. Nature 432, 980-987 (2004).

31. Serrano, M., Hannon, G. J. \& Beach, D. A new regulatory motif in cell-cycle control causing specific inhibition of cyclin D/CDK4. Nature 366, 704-707 (1993).

32. Duesbery, N. S. et al. CENP-E is an essential kinetochore motor in maturing oocytes and is masked during mos-dependent, cell cycle arrest at metaphase II. Proc. Natl Acad. Sci. USA 94, 9165-9170 (1997).

33. Boudeau, J., Sapkota, G. \& Alessi, D. R. LKB1, a protein kinase regulating cell proliferation and polarity. FEBS Lett. 546, 159-165 (2003).

34. Liu, W. et al. LKB1/STK11 inactivation leads to expansion of a prometastatic tumor subpopulation in melanoma. Cancer Cell 21, 751-764 (2012).

35. Finn, R. S. et al. PD 0332991, a selective cyclin D kinase $4 / 6$ inhibitor, preferentially inhibits proliferation of luminal estrogen receptor-positive human breast cancer cell lines in vitro. Breast Cancer Res. 11, R77 (2009).

36. Yoshida, A. \& Diehl, J. A. CDK4/6 inhibitor: from quiescence to senescence. Oncoscience 2, 896-897 (2015).

37. Weinberg, R. A. The retinoblastoma protein and cell cycle control. Cell $\mathbf{8 1}$, 323-330 (1995).

38. Ji, H. et al. LKB1 modulates lung cancer differentiation and metastasis. Nature 448, 807-810 (2007)

39. Lizcano, J. M. et al. LKB1 is a master kinase that activates 13 kinases of the AMPK subfamily, including MARK/PAR-1. EMBO J. 23, 833-843 (2004).

40. Nakada, D., Saunders, T. L. \& Morrison, S. J. Lkb1 regulates cell cycle and energy metabolism in haematopoietic stem cells. Nature 468, 653-658 (2010).

41. Yang, Y. et al. Spatial-temporal lineage restrictions of embryonic p63(+) progenitors establish distinct stem cell pools in adult airways. Dev. Cell 44, 752-761 e754 (2018).

42. Sakamoto, K., Goransson, O., Hardie, D. G. \& Alessi, D. R. Activity of LKB1 and AMPK-related kinases in skeletal muscle: effects of contraction, phenformin, and AICAR. Am. J. Physiol. Endocrinol. Metab. 287, E310-E317 (2004).
43. Sun, Y., Connors, K. E. \& Yang, D. Q. AICAR induces phosphorylation of AMPK in an ATM-dependent, LKB1-independent manner. Mol. Cell. Biochem. 306 239-245 (2007).

44. Muller, J., Ory, S., Copeland, T., Piwnica-Worms, H. \& Morrison, D. K. C-TAK1 regulates Ras signaling by phosphorylating the MAPK scaffold, KSR1. Mol. Cell 8, 983-993 (2001).

45. Muller, J., Ritt, D. A., Copeland, T. D. \& Morrison, D. K. Functional analysis of CTAK1 substrate binding and identification of PKP2 as a new C-TAK1 substrate. EMBO J. 22, 4431-4442 (2003).

46. Chang, L. \& Karin, M. Mammalian MAP kinase signalling cascades. Nature $\mathbf{4 1 0}$ 37-40 (2001).

47. Zhang, W. \& Liu, H. T. MAPK signal pathways in the regulation of cell proliferation in mammalian cells. Cell Res. 12, 9-18 (2002).

48. Lubman, O. Y., Korolev, S. V. \& Kopan, R. Anchoring notch genetics and biochemistry; structural analysis of the ankyrin domain sheds light on existing data. Mol. Cell 13, 619-626 (2004).

49. Meloche, S. \& Pouyssegur, J. The ERK1/2 mitogen-activated protein kinase pathway as a master regulator of the G1- to S-phase transition. Oncogene 26, 3227-3239 (2007)

50. Ito, T. et al. Basic helix-loop-helix transcription factors regulate the neuroendocrine differentiation of fetal mouse pulmonary epithelium. Development 127, 3913-3921 (2000).

51. Danielian, P. S. et al. E2f4 is required for normal development of the airway epithelium. Dev. Biol. 305, 564-576 (2007).

52. Ma, L., Quigley, l., Omran, H. \& Kintner, C. Multicilin drives centriole biogenesis via E2f proteins. Genes Dev. 28, 1461-1471 (2014).

53. Zhao, $\mathrm{H}$. et al. The Cep63 paralogue Deup1 enables massive de novo centriole biogenesis for vertebrate multiciliogenesis. Nat. Cell Biol. 15, 1434-1444 (2013)

54. Kim, S., Ma, L., Shokhirev, M. N., Quigley, I. \& Kintner, C. Multicilin and activated E2f4 induce multiciliated cell differentiation in primary fibroblasts. Sci. Rep. 8, 12369 (2018).

55. Harfe, B. D. et al. Evidence for an expansion-based temporal Shh gradient in specifying vertebrate digit identities. Cell 118, 517-528 (2004).

56. Muzumdar, M. D., Tasic, B., Miyamichi, K., Li, L. \& Luo, L. A global doublefluorescent Cre reporter mouse. Genesis 45, 593-605 (2007).

57. Han, $\mathrm{H}$. et al. Inducible gene knockout of transcription factor recombination signal binding protein-J reveals its essential role in T versus $B$ lineage decision. Int. Immunol. 14, 637-645 (2002).

58. Madisen, L. et al. A robust and high-throughput Cre reporting and characterization system for the whole mouse brain. Nat. Neurosci. 13, 133-140 (2010).

59. Qiu, X. et al. Single-cell mRNA quantification and differential analysis with Census. Nat. Methods 14, 309-315 (2017). 